\title{
The role of Fever in Covid-19
}

\section{K. M. Yacob}

Chief Physician, Marma Health Centre, Kerala, India.

Corresponding Author: K. M. Yacob Chief Physician, Marma Health Centre, Kerala, India.

Received date: December 30, 2020; Accepted date: January 04, 2021; Published date: March 17, 2021

Citation: K. M. Yacob (2021) The role of Fever in Covid-19 J, Clinical Medical Reviews and Reports. 3(2); DOI:10.31579/2690-8794/062

Copyright: (C2021, K. M. Yacob, This is an open access article distributed under the Creative Commons Attribution License, which permits unrestricted use, distribution, and reproduction in any medium, provided the original work is properly cited.

\section{Introduction}

We are living with a lot of diseases related to fevers with different names like Chikungunya fever, West Nile fever, rheumatic fever, Relapsing fever, Rat-bite fever (sodoku), Haver hill fever, Dengue fever, Typhoid fever, Scarlet fever, Viral hemorrhagic fever, etc.

In COVID -19 up to $85 \%$ of patients have no fever. Why up to $80 \%$ of patients with the COVID -19 (SARS-CoV-2) virus have no fever? Is it because there is no need for the care of fever or is it because the disease is free of signals or symptoms?

\section{Are there different types of fevers? Or is it just a fever?}

There is only one Fever. Definition, diagnosis, and treatment of fever are the same. The symptoms, signs, signals, and actions of the disease are different but the symptoms, signs, signals, and actions of fever are the same. That means there is a scientific basis, which is common for all these diseases related to fever and can be used to explain the secret of all disease-related with fever.

Let us examine the Present State of Fever. One medical book says.

"Our understanding of the neural basis of thermoregulation and fever is still rudimentary".

How can fever be treated with rudimentary knowledge?

The fact that the patient does not know that the fever is being treated by rudimentary knowledge is the basis for the survival of the physician.

A scientific approach is necessary to evaluate and treat fever. My research is to find out the purpose of the rise of temperature in the case of fever.

The following 4 questions need to be answered to know about fever

1. What are the components required to develop a fever and hyperthermia?

2. What are the components required to curing fever and hyperthermia?

3. What is the purpose of the temperature of a fever?

4. The heat energy used for which activities in a fever and hyperthermia?

The above 4 questions are not answered in any medical book.

\section{Necessary components required to develop a fever and hyperthermia}

The right ratio of components like inflammation, pyrogens are required to develop fever-like good health.

Inflammation and pyrogens never cause hyperthermia. All that is needed for hyperthermia is uncontrolled heat only.

\section{Necessary components required to cure fever and hyperthermia}

For curing fever we should know what the components necessary to develop a fever are. Disperse the correct ratio of components of fever will decrease the intensity of fever. By removing the correct ratio of components of fever will cure the fever:-If the inflammation is reduced, the fever will go away. The only way to change hyperthermia is to water and reduce the heat. Hyperthermia does not go away because the inflammation is reduced like a fever.

\section{What is the Purpose of the fever In Covid -19?}

When the disease made by the SARS COV-2 virus (bacteria, fungi, venom, horror scene, and horror dream, etc..., in other diseases) becomes a threat to life or organs blood circulation decreases, Temperature of fever will emerge to increase prevailing essential blood circulation. And it acts as a protective covering of the body to sustain life. It is an adaptation.

In all diseases which decrease essential blood circulation and temperature, the fever will emerge to Increase essential blood circulation and temperature. There is no way other than this to increase prevailing essential blood circulation for a sensible and discreet immune system to protect the life or organ.

Modern science says that fever is an immune response against infection. If fever is an immune response against infection, the temperature of fever is not a surplus temperature. It is not supposed to be eliminated from the body. If we try to eliminate the immune response against infection, the infection will increase. It is dangerous to the immune system. It is necessary to protect the immune system. To increase essential blood circulation, never allow body temperature to lose via the atmosphere.

A brooding hen's body temperature increase like that during fever to increase essential blood circulation in the body. The brooding hen does not eat any food. Brooding hen's Food is its heat, which helps to convert fat into energy.

Many people have a misconception that fever should be Cured (changed). It is not the fever that needs to be cured (changed), it is the disease or cause of the disease that needs to be cured. Fever is an adaptation only. Fever can be compared to our soldiers. Sometimes our patriotic soldiers may be defeated, surrendered, or even die. It is not due to the anti-national activity of our soldiers.

\section{New fever Definition}


If essential blood circulation decreases to organs, fever is a sensible and discreet action of the immune system to increase essential blood circulation as a self-defense mechanism of the body to sustain the life or organ.

By this definition, we can answer any questions about fever.

If we deviate from this Definition, we will not be able to give a correct answer to even a single question.

\section{Why does not everyone with the disease have a fever?}

3 reasons.

1. Virus, bacteria, fungi, etc do not cause fever in everyone because the causes of the disease do not decrease essential blood circulation to organs.

2. The body does not need fever protection.

3. The immune system does not have the energy to produce the temperature of fever as in AIDS.

\section{How can create Fever and hyperpyrexia and fits within hours?}

In people with tonsillitis, children, and the elderly using Ice water, we can create fever and hyperpyrexia within a few hours. With continued use, we can cause death in them. Thalaikoothal is the best example. Thalaikoothal is the traditional practice of suicide (killing of the elderly) or involuntary euthanasia, by their own family members, observed in some parts of southern districts of Tamilnadu, State of India. This is a process in which an extensive bath is given to an elderly person before the crack of dawn. He or she is given several glasses of cold tender coconut water and a head massage with cold water. By doing this the temperature of the body falls suddenly and causing high fever, fits which leads to the death of the old aged person within 1-2 days. (Reference 9, $10,11)$.

Similarly in Covid patients, using sponging with Ice water, we can create fever and hyperpyrexia within a few hours. With continued use, we can cause death in them.

\section{Thought-provoking questions and answers}

1. If pouring cold water on the body can reduce body heat and cause fever, hyperpyrexia in Thalaikoothal, cannot any disease cause fever and hyperpyrexia by pouring water on the body? Answer: Can.

2. Is there any difference between a fever (elevated temperature in the hypothalamus) caused by Thalaikoothal and other illnesses?

Answer: No.

3. Is there any difference between Fever, fits, and death due to heat loss in Thalaikoothal and Fever, fits, and death due to heat loss in Covid19 ?

Answer: No.

4. Is there any difference between Covid -19 fever treatment and Thalaikoothal fever treatment?

Answer: No.

5. Is there any difference between Covid -19 fever treatment and any other fever treatment?

Answer: No.

6. Is Conservative fever treatment suitable for Thalaikoothal?

Answer: No
7. In Thalaikoothal what are the necessary ingredients to cure hyperpyrexia?

Answer: To increase blood circulation, and to decrease inflammation heat supplementation is necessary. And prevent heat loss from the body.

8. If fever with Thalaikoothal can be cured with heat, can fever with any diseases not be cured with heat?

Answer: can

9. If watering the body can reduce body temperature and cause fever, then watering again can reduce the temperature of the body and cause fits and death as in Thalaikoothal, if modern fever treatment can be done by sponging or immersing the body in ice water in hyperpyrexia, can it not cause fits and death by reducing the body temperature?

Answer: Can.

Most medical books say that patients with hyperpyrexia should be immediately immersed in ice water regardless of the cause of the disease.

10. According to this, in patients with hyperpyrexia due to Thalaikoothal, does immersion in ice water cause death?

Answer: There will be death.

11. Isn't science the same in both?

Answer: Yes.

12. Will existing fever treatment reduce Covid death?

Answer: No

13. Did any patient die without developing a fever in Covid-19 and without heat reduction treatment for that fever?

Answer: Unlikely to occur.

How unscientific it is to say that prolonged exposure to water can cause fever and that fever can be cured by sponging it in water. Fever is measured with a thermometer, and hyperthermia is also measured with the same thermometer. This is purely unscientific. The basic elements necessary for a scientific treatment is not provided in fever treatment.

\section{How can it be proven that the fever of covid -19 is to increase essential blood circulation?}

1. If we ask any type of question-related to fever by assuming that the temperature of fever is to increase essential blood circulation we will get a clear answer. If we deviate from this, we will not be able to give a correct answer to even a single question.

2. If we do any type of treatment by assuming that the temperature of fever is to increase essential blood circulation, the body will accept, at the same time body will resist whatever treatment to decrease blood circulation.

3. If we measure the heat energy used for which activities in fever, we will know the purpose of the temperature of fever.

It is astonishing that modern science has not been able to examine what actions with the heat energy of fever, despite its advanced development.

No further evidence is required to prove the temperature of fever is to increase essential blood circulation. Apart from this, what kind of evidence is needed to prove that the temperature of the fever can increase blood circulation? 
Let me know new parameters to prove the purpose of the temperature of fever, we can prove that the temperature of fever is to increase essential blood circulation with those parameters.

During fever if we take a steam bath, fits never happens, blood circulation never decreases, the skin never shrinks as in fever.

\section{Science of healing Fits}

Regain consciousness even before the temperature subsides. When a fainted patient lie on the floor the vertical height between the heart and brain decreases. So blood circulation increases to the brain, and so fits is cured even before temperature subsides. Many people misunderstand that regained consciousness is due to the reduction of temperature.

\section{Shivering is to increase blood circulation.}

Hyper Pyrexia's temperature is below 42 degrees centigrade, only a 4degree increase in body temperature. If a 4-degree increase in temperature occurs there is no history to show that shivering occurs. If disease increases, blood circulation decreases. Shivering is not due to an increase in fever, but due to the increase of disease, decrease of energy, immunity power, strength, essential blood circulation, etc. shivering helps to increase heat, then essential blood circulation will increase. Fever and Shivering are protective actions according to the aim and target of the immune system.

If the body produces an excess amount of heat/ energy/ force/strength the body will never shiver. During fever, one shivers due to a lack of enough temperature in the shivering area. In other words, if a body has enough temperature to maintain its normal body temperature it will not shiver. The decrease in essential temperature to sustain life is the cause of shivering. In fever condition, how much essential temperature is decreased in the body, the corresponding temperature is produced to generate a counterbalance of the decreasing essential temperature.

\section{Animal behaviour - Advantage of instinct}

Buffaloes do not try to enter/lie in the water nor drink it during fever.

Buffalos manage body temperature by immersing their body in water and drink it. But when they are having a fever, they do not try to enter the water nor drink it.

\section{Method of increasing}

\section{Blood circulation and eliminating fever}

Look at the message from our body and act accordingly. The only proper treatment for fever is to increase essential blood circulation. Without an increase in temperature, blood circulation never increases without an increase in essential blood circulation, the fits will never change. To increase essential blood circulation never allow body temperature to lose via the atmosphere and apply extra heat from outside and inside to the body. It will help the immune system to fight against infection. Blanket, Steam bath, hot sandbag or thermal heat pad, Hot pepper water, Hot drinks can be used.

\section{What happens if a person with fever gets heat from outside of the body?}

The heat generated by our immune system combines with the heat we receive from outside the body to increase essential blood circulation. Then our immune system will stop producing heat that is made to increase essential blood circulation. Thus our body receives heat from outside to increase essential blood circulation without using its energy.

Heat is the best way to reduce inflammation and increase blood circulation faster
During fever, $99 \%$ of diseases or patients show inflammation. Apply heat from outside and inside to the body, blood circulation, and inflammationrelated fever and its signals, symptoms, signs, and actions will decrease. Then essential blood circulation increases, inflammation decreases, digestion increases, body pain diminishes.

\section{Like and accept the hotness.}

When we apply heat from outside and inside to the body, all the signals, symptoms of fever, like and accept the hotness according to the directions and commands of the Immune system. If fever is an immune response against infection, our body likes and accepts hotness, at the same time our body dislikes and hate temperature reducing methods. If fever is an immune response against infection, the temperature of fever is not a surplus temperature. If we reduce the temperature by water sponging, the infection will increase and it may be dangerous.

Fever is not a part of the disease, it is a part of the self-defense mechanism of the body, and it is an adaptation. A disease does not require fever but, at the same time, a fever requires a disease. Both disease and fever are different. We cannot include body destructing disease and protecting fever in the same group.

\section{Conservative fever treatments and their effects.}

Fever

\section{Treatments- Role sponging and paracetamol.}

Whatever happens to a brooding hen and its egg if sponged with water, the same happens to a fever patient if the temperature is decreased by sponging.

The researchers found that the glutathione level was lower when a Single dose of paracetamol was used.8. This may increase the inflammation level.

Removal of the temperature of fever by sponging or paracetamol is not helpful for curing disease.

It is believed that the doctor will save the patient in the treatment of fever, but the doctor becomes the executioner who kills the person.

All conservative treatments for fever not only don't make a radical cure of the feverish condition but also further decrease essential blood circulation and increase inflammation and lead the body to more dangers.

If the temperature is decreased by sponging or paracetamol, disease or cause of disease or cause of fever is never decreased. It is proved that fever medicines are more dangerous than the disease, and fever treatments are more dangerous than medicines for fever.

\section{Is there any base for conservative fever treatment?}

1. All treatments for fever are based on the belief that The fever temperature between 38 to 41 is harmful to the brain and body. At the same time there no concrete evidence to this belief 1

2. The purpose of the rise of temperature is not known 2 .

Without knowing the purpose of the temperature of fever and without examining what action was taken with the heat energy of the fever, what science can be used to say that the fever is dangerous?

\section{There is only one way to reduce covid deaths.}

The best way to reduce the Covid death rate is to Prohibit Thalaikoothal modern conservative fever treatment. There is no other way than this. 


\section{Importance of the findings of the purpose of the temperature of} fever in Covid-19

Immediate relief from fever and body pain. Life-saving discovery. A single magic answer to every fever-related questions. If medicines are prepared according to the purpose of the temperature of fever any country can guide the world in the cure for fever.

\section{Conclusion}

When the disease becomes a threat to life or organs, the temperature of fever in Covid -19 is to increase essential blood circulation. Fits, delirious, or damage of brain cells are not caused when an increase in the temperature of fever takes place. It is due to a decrease in blood circulation to the brain.

Apply extra heat from outside and inside to the body will increase Blood Circulation. Fever treatment should be revised according to the sensible and discreet action of the immune system. It is wonderful that a physician having years of experience in fever treatment cannot understand the actual purpose of fever. I have developed 8000 Affirmative cross checking questions. It proves that fever is to increase essential blood circulation.

\section{Reference}

1. Nelson Textbook of Pediatrics 20th edition.

2. Pharmacology and pharmacotherapeutics -R.S.Satoskar, S.D.Bhandarkar, Nirmala N.Rege- Revised XIV edition, p.159, 160, 163, 170).

3. National Medical Series for Independent Study-NMS Medicine 4th edition, Edited by Allen R. Myers, MD. page430.

4. Journal of Applied Medicine, Dr.M.McD Fisher and Dr. Raper, page 188, 1988 March

5. Davidsons Principles and practice of medicine_22Ed.

6. Text book of Medical Physiology-Guyton and Hall,11 th edition

7. Berman's Pediatric Decision Making (5th edition) 2011.

8. Dr.John T. McBride, PEDIATRICS Volume 128, Number 6, December 2011 (ISSN Numbers: Print, 0031-4005; Online, 10984275).

9. www.ncbi.nlm.nih.gov/pmc/articles/PMC6504736

10. https://en.wikiprdia.org/wiki/Thalaikoothal

11. https://www.researchgate.net/publication/269404983_Thalaikoot hal_The_Practice_of_Euth anasia_in_the_Name_of_Custom 